\title{
Association of MUTYH and colorectal cancer
}

\author{
A Tenesa ${ }^{*, 1,2}$, H Campbell ${ }^{1,3}$, R Barnetson ${ }^{1,2}$, M Porteous ${ }^{1,4}$, M Dunlop ${ }^{1,2}$ and SM Farrington ${ }^{*, 1,2}$ \\ 'Colon Cancer Genetics Group, University of Edinburgh, Western General Hospital, Crewe Road, Edinburgh, UK; ${ }^{2}$ MRC Human Genetics Unit, Western \\ General Hospital, Crewe Road, Edinburgh, UK; ${ }^{3}$ Public Health Sciences, University of Edinburgh, Teviot Place, Edinburgh EH8 9AG, UK; ${ }^{4}$ Clinical Genetics \\ Department, University of Edinburgh, Edinburgh EH4 2XU, UK
}

Mutations in the MUTYH gene have been reported to be associated with increased risk of developing colorectal cancer. In this study, we confirmed this association using original data on 928 colorectal cancer cases and 845 healthy controls from Scotland. We then conducted a meta-analysis from published data on the association between mutations at MUTYH and colorectal cancer risk. We show for the first time a small but significant mono-allelic effect with a genotype relative risk (GRR) of I.27 (95\% confidence interval (Cl): I.0I- I.6I), and confirm and give a more precise estimate of the strong bi-allelic effect with an estimated GRR of I I7 (95\% Cl: 74-184). This study underscores the need for large sample sizes in order to identify small gene effects when the disease allele frequency is low.

British Journal of Cancer (2006) 95, 239-242. doi:I 0.1038/sj.bjc.6603239 www.bjcancer.com

Published online 27 June 2006

(c) 2006 Cancer Research UK

Keywords: MUTYH; BER; MYH; colorectal cancer

DNA repair genes are strong candidate cancer susceptibility genes (Ames and Gold, 1991; Yoshimura et al, 2003). One such gene is $M U T Y H$, which together with OGG1 and MTH1 is a key component of the base-excision repair (BER) pathway. The main function of the BER pathway is to repair DNA oxidative damage caused by aerobic metabolism. Hence, MUTYH has raised much interest among those trying to unravel the genetic contribution to cancer risk (Al-Tassan et al, 2004; Tao et al, 2004; Farrington et al, 2005).

MUTYH has been associated with multiple colorectal adenomas (Al-Tassan et al, 2002) and with colorectal adenocarcinomas (Croitoru et al, 2004; Farrington et al, 2005). Here, we present a replication study in the Scottish population and a meta-analysis of published case-control data on MUTYH and colorectal cancer. Both types of study are important, because genetic association studies involving complex traits are frequently inconclusive owing to the difficulty of isolating the causal variant effect from other confounding factors. Hence, replication of the original findings is needed to support the validity of the association. Nevertheless, this might not always be possible, as unrealistically large follow-up studies would be required to detect small gene effects and in such instances the meta-analysis of published data may clarify the credibility of any inconclusive association.

\footnotetext{
* Correspondence: Dr A Tenesa or Dr S Farrington, Colon Cancer Genetics Group, School of Molecular and Clinical Medicine, The University of Edinburgh, 4th Floor, MRC Human Genetics Unit, Western General Hospital, Crewe Road South, Edinburgh EH4 2XU, UK. E-mails: albert.tenesa@ed.ac.uk, susan.farrington@hgu.mrc.ac.uk Received 19 January 2006; revised I June 2006; accepted 6 June 2006; published online 27 June 2006
}

\section{MATERIALS AND METHODS}

\section{Replication data}

We previously reported an association between MUTYH and colorectal cancer in a population-based study of 2239 cases (with histologically confirmed adenocarcinomas) and 1845 controls in Scotland. MUTYH homozygous mutation carriers had a 90 -fold excess risk, whereas heterozygous carriers had no significant increased risk compared to wild-type homozygous on the overall cohort (Table 1), although there was a significant heterozygous effect in the late-onset cohort. Here, we extend this populationbased study to include a further 928 colorectal cancer cases and 845 healthy controls. These samples were collected from the general population of Scotland. Cases were ascertained through all surgical Units in Scotland dealing with colorectal cancer. All cases had histologically confirmed adenocarcinoma of the colon or rectum. Blood DNA samples were obtained from the patients after fully informed consent. The study is subject to approvals from the Multi Centre Research Ethics Committee and all relevant Local Ethics Research Committee, as well as approval from NHS R\&D Management for every participating hospital.

The two most common MUTYH variants in the Scottish population, Y165C and G382D, were genotyped following methods described previously (Farrington et al, 2005). Genotypes were coded as MM if the person was an Y165C/Y165C or G382D/G382D homozygote or Y165C/G382D or other compound heterozygote (detected by sequencing the entire coding region of the second allele of G382D/Y165C heterozygotes); as WM if the person was an Y165C or G382D heterozygote and otherwise as WW. All cases and $67 \%$ of controls were screened for a second mutation if they happened to be an Y165C or G382D heterozygote. Owing to the low frequency of compound heterozygotes other than Y165C/G382D in cases (1/12 in our original report) this is expected to have little effect on the analysis that follows. 


\section{Meta-analysis data}

In order to identify all relevant studies for the meta-analysis of the effect of MUTYH on colorectal cancer risk, we searched ISI Web of SCIENCE (http://wok.mimas.ac.uk/) and PUBMED (http:// www.ncbi.nlm.nih.gov/entrez/query.fcgi) for the relevant literature references. We found 55 studies (searching for 'MYH and colorectal cancer'), but only eight studies contained data that met our inclusion criteria and thus were relevant to this metaanalysis. These criteria were that patients had confirmed colorectal adenocarcinoma (i.e. we excluded studies only based on the multiple adenoma phenotype), and that the study reported genotype data for cases and controls (Table 2). Table 3 includes information on how the samples for each of the studies included in the meta-analysis were collected. Individuals reported to have two defects at MUTYH in the original report were classified as MM (defects were considered pathogenic only if there was published evidence of their pathogenicity), those with one defect as WM and those with no detected defect as WW. In total, we assembled data on 13449 people: 7273 colorectal cancer patients and 6176 population-based controls.

\section{Statistical methods}

The association of MUTYH with colorectal cancer was tested by means of a standard $\chi^{2}$ test with 2 degrees of freedom, and genotype relative risks (GRRs) were estimated as described previously (Farrington et al, 2005).

We used the metabin option from the meta package of the $R$ software to perform the meta-analysis. We used the summary measure relative risk and the inverse variance weighting to pool studies. For mathematical reasons, cells with zero frequencies were

Table I Numbers of cases and controls, GRR and empirical 95\% Cl from the population-based study in Scotland: data from the original report, the replication study and from the combined data $^{a}$

\begin{tabular}{|c|c|c|c|c|c|c|}
\hline \multirow[b]{2}{*}{ Genotype } & \multicolumn{2}{|c|}{ Farrington et al (2005) $(P=0.00 \mathrm{I})$} & \multicolumn{2}{|c|}{ Replication $(P=0.09)$} & \multicolumn{2}{|c|}{ All together $(P=0.0006)$} \\
\hline & No. of cases/controls & GRR (95\% Cl) & No. of cases/controls & GRR (95\% Cl) & No. of cases/controls & GRR (95\% Cl) \\
\hline Total & $2217 / 1822$ & & $928 / 845$ & & $3145 / 2667$ & \\
\hline
\end{tabular}

${ }^{a} \mathrm{GRR}$ is the genotype relative risk, $\mathrm{Cl}$ is the confidence interval and $P$ is the significance level for the test of association using a $\chi^{2}$ test with 2 degrees of freedom.

Table 2 Association between MUTYH and colorectal cancer: review and meta-analysis of published data

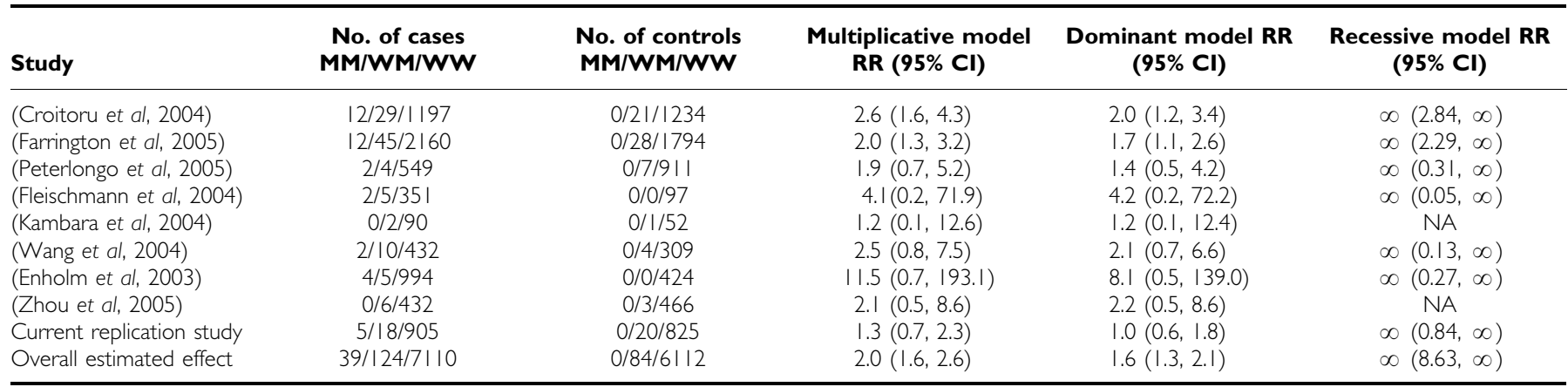

$\mathrm{Cl}=$ confidence interval; $\mathrm{NA}=$ not available; $\mathrm{RR}=$ relative risk. $\mathrm{M}$ vs $\mathrm{W}$ allele for the multiplicative model, $\mathrm{MM}$ and $\mathrm{MW}$ vs $\mathrm{WW}$ for the dominant model and $\mathrm{MM}$ vs $\mathrm{MW}$ and

WW for the recessive model. The recessive model RR confidence intervals were estimated using Fisher's exact test.

Table 3 Information on the collection of samples of the studies included in the meta-analysis

\begin{tabular}{|c|c|c|c|c|}
\hline Study & Cases description & Population & Controls description & Population \\
\hline (Croitoru et al, 2004) & Prospective series & Regional Canadian & Age and sex matched & Same region of Canada \\
\hline $\begin{array}{l}\text { (Farrington et al, 2005) and } \\
\text { replication }\end{array}$ & Prospective series & Scottish population & Age, sex and region matched & Scottish population \\
\hline (Peterlongo et al, 2005) & Consecutive series & Regional USA & $\begin{array}{l}\text { Chosen from } 17000 \text { cohort } \\
\text { study as cancer free and age } \\
\text { matched }\end{array}$ & $\begin{array}{l}\text { Same region USA and } \\
\text { ethnically matched. }\end{array}$ \\
\hline (Fleischmann et al, 2004) & Unrelated retrospective series & Three regions of UK & $\begin{array}{l}\text { Spouses of individuals from } \\
\text { another cancer study }\end{array}$ & No information \\
\hline (Kambara et al, 2004) & Sporadic series & Regional Australia & Healthy blood donors & Same region of Australia \\
\hline (Wang et al, 2004) & No information & Regional USA & $\begin{array}{l}\text { Individuals negative by } \\
\text { colonoscopy }\end{array}$ & Same region of USA \\
\hline (Enholm et al, 2003) & Systematic series & Finnish population & Healthy blood donors & No information \\
\hline (Zhou et al, 2005) & Sporadic series & Three regions of Sweden & Healthy blood donors & Stockholm region \\
\hline
\end{tabular}


assumed to be 0.5 (as defaulted by the meta package). For the recessive model with zero cell counts, we estimated exact confidence intervals (CIs) using Fisher's exact test.

\section{RESULTS}

\section{Replication study}

Table 1 shows the results obtained in this replication study, from our previously published data and from the combined data set. The replication study showed a significant homozygous MM effect $(P<0.05)$, and the results were suggestive of an association with the MUTYH gene as a whole $(P=0.09)$. Combining these data with our previously published data revealed a highly significant association with MUTYH $(P=0.0006)$. However, a heterozygous effect was not detected (Peterlongo et al, 2005; Tenesa et al, 2005).

\section{Meta-analysis}

First, we tested the overall association of the gene with colorectal cancer assuming three plausible genetic models: multiplicative, dominant and recessive. We found that the association was highly significant $(P \leqslant 0.0004)$ under the three genetic models considered. We did not find significant heterogeneity between studies under any of the models considered. The value of $I^{2}$, which quantifies the level of heterogeneity on a continuous scale (Higgins and

Table 4 MUTYH genotype effect on colorectal cancer risk: review and meta-analysis of published data

\begin{tabular}{llc}
\hline Study & $\begin{array}{c}\text { Heterozygous } \\
\text { (WM) effect } \\
\text { RR (95\% Cl) }\end{array}$ & $\begin{array}{c}\text { Homozygous } \\
\text { (MM) effect }^{\mathbf{a}} \\
\text { RR (95\% Cl) }\end{array}$ \\
\hline (Croitoru et al, 2004) & $1.4(0.8,2.5)$ & $\infty(2.86, \infty)$ \\
(Farrington et al, 2005) & $1.3(0.8,2.1)$ & $\infty(2.30, \infty)$ \\
(Peterlongo et al, 2005) & $0.9(0.3,3.2)$ & $\infty(0.31, \infty)$ \\
(Fleischmann et al, 2004) & $3.1(0.2,54.7)$ & $\infty(0.05, \infty)$ \\
(Kambara et al, 2004) & $1.2(0.1,12.4)$ & $\mathrm{NA}$ \\
(Wang et al, 2004) & $1.8(0.6,5.6)$ & $\infty(0.14, \infty)$ \\
(Enholm et al, 2003) & $4.7(0.3,84.7)$ & $\infty(0.28, \infty)$ \\
(Zhou et al, 2005) & $2.2(0.5,8.6)$ & $\mathrm{NA}$ \\
Current replication study & $0.8(0.4,1.6)$ & $\infty(0.83, \infty)$ \\
Overall effect & $1.3(1.0,1.7)$ & $\infty(8.63, \infty)$ \\
\hline
\end{tabular}

$\mathrm{Cl}=$ confidence interval; $\mathrm{NA}=$ not available; $\mathrm{RR}=$ relative risk. ${ }^{\mathrm{a}} 95 \% \mathrm{Cl}$ estimated using exact methods (Fisher exact test).
Thompson, 2002), was 0\% (95\% CI: $0.0-48.7), 0 \%$ (95\% CI: $0.0-$ $45.9)$ and $0 \%$ (95\% CI: $0.0-39.8)$ for the multiplicative, dominant and recessive model, respectively. Therefore, we assumed that the effect was the same across studies and used a fixed effects model. Note, however, that using a random effects model gave identical results.

Next, we tested whether there was a homozygous (MM) and heterozygous (WM) gene effect (Table 4). The overall homozygous effect was highly significant $(P=0.0004)$, whereas the overall heterozygous effect was almost significant $(P=0.09)$.

To address the possibility that methodological differences across studies may have influenced these results, we pooled all available data described in Table 2 and estimated the GRRs as before (Farrington et al, 2005). We considered this was justified, as there was no statistically significant heterogeneity among studies. We performed the analyses both for all the 13449 samples (data set 1) and separately for the 7657 samples not generated by our group (data set 2). The 95\% CI for the GRR estimates in the two data sets overlapped both for MM and WM individuals. Data set 1 gave GRR for the MM and WM equal to 117 (95\% CI: 74-184) and 1.27 (95\% CI: $1.01-1.61)$, respectively. The overall MUTYH variant frequency was $0.34 \%$. Similarly, data set 2 gave GRR for the MM equal to 207 (95\% CI: $109-415)$ and 1.47 (95\% CI: $1.05-2.12)$ for WM. Both data sets showed a highly significant $(P<0.001)$ homozygous effect and a heterozygous effect of borderline statistical significance $(P<0.05)$.

Finally, we tested whether there were differences in the effect of Y165C and G382D mutations (Table 5). Estimates of the monoallelic effect were similar and not significantly different at the $5 \%$ level. However, the bi-allelic effect was much larger for Y165C carriers than for G382D carriers, although this difference was not statistically significant.

\section{DISCUSSION}

This study confirms, in a combined data set of over 7000 cases across several study populations, that the association between variants in the MUTYH gene and colorectal cancer risk is valid. Bi-allelic inactivation of the gene conferred a very large increase in risk $(\mathrm{GRR}=117)$ supporting its causal role in colorectal cancer, whereas mono-allelic inativation of the gene conferred a moderate increase in risk $(\mathrm{GRR}=1.3)$. Confidence intervals for the estimate of the risk associated with germline bi-allelic defects were wide, even though the total sample size was over 13000 individuals. This effect might be substantially overestimated if the proportion of compound heterozygotes in the control sample was equal to the case sample (i.e. 1/46), but we believe this is highly unlikely as we

Table 5 Counts of G382D and YI65C mutations reported in the published data and GRR

\begin{tabular}{|c|c|c|c|c|}
\hline \multirow[b]{2}{*}{ Study } & \multicolumn{2}{|c|}{ G382D } & \multicolumn{2}{|c|}{ YI65C } \\
\hline & Cases & Controls & Cases & Controls \\
\hline (Croitoru et al, 2004) & $4 / 21 / 1197$ & $0 / 17 / 1234$ & $2 / 8 / 1197$ & $0 / 4 / 1234$ \\
\hline (Farrington et al, 2005) & $8 / 31 / 2160$ & $0 / 20 / 1794$ & $0 / 14 / 2160$ & $0 / 8 / 1794$ \\
\hline (Peterlongo et al, 2005) & |/2/549 & $0 / 5 / 911$ & 0/2/549 & $0 / 2 / 911$ \\
\hline (Fleischmann et al, 2004) & $|/ 4 / 35|$ & 0/0/97 & $0 / \mid / 351$ & 0/0/97 \\
\hline (Kambara et al, 2004) & 0/2/90 & 0/0/52 & 0/0/90 & $0 / 1 / 52$ \\
\hline (Wang et al, 2004) & $0 / 5 / 432$ & 0/2/309 & 1/5/432 & 0/2/309 \\
\hline (Enholm et al, 2003) & | /4/994 & $0 / 0 / 424$ & 0/1/994 & $0 / 0 / 424$ \\
\hline (Zhou et al, 2005) & $0 / 2 / 432$ & $0 / 1 / 466$ & $0 / 4 / 432$ & $0 / 2 / 466$ \\
\hline Current replication study & 2/15/905 & $0 / 14 / 825$ & 2/3/905 & 0/6/825 \\
\hline Overall numbers & $17 / 86 / 7 \mid 10$ & $0 / 59 / 6112$ & $5 / 38 / 7 \mid 10$ & $0 / 25 / 6112$ \\
\hline GRR (MM/WM/WW) & \multicolumn{2}{|c|}{$|03.63 / 1.26 /|$} & \multicolumn{2}{|c|}{ |68.82/|.31/| } \\
\hline GRR 95\% Cl (MM/WM/WW) & \multicolumn{2}{|c|}{$(55.26-\mid 88.61) /(0.96-1.68) /(1.00-1.00)$} & \multicolumn{2}{|c|}{$(48.77-438.31) /(0.86-2.06) /(1.00-1.00)$} \\
\hline
\end{tabular}

$\mathrm{Cl}=$ confidence interval; $\mathrm{GRR}=$ genotype relative risk. 
resequenced 32 (i.e. two-third) of our heterozygote controls and did not find any compound heterozygotes.

Similarly, mono-allelic defects are of borderline statistical significance. This underscores the important role of meta-analyses of large data sets from well-conducted studies to properly interpret these relationships. It is difficult to assess whether differences in the screening method employed by different studies might explain the small heterozygous effect (i.e. not all studies did a systematic screening of heterozygous individuals in order to discard other possible disease variants), but additional support from other studies (Jenkins et al, 2006) that used different methods and samples suggest that the small heterozygous effect is real. Hidden compound heterozygotes would further increase the numbers required to assess whether there is indeed a heterozygous effect because the heterozygous effect would be even smaller.

\section{REFERENCES}

Al-Tassan N, Chmiel NH, Maynard J, Fleming N, Livingston AL, Williams GT, Hodges AK, Davies DR, David SS, Sampson JR, Cheadle JP (2002) Inherited variants of MYH associated with somatic G:C- $>$ T:A mutations in colorectal tumors. Nat Genet 30: 227-232

Al-Tassan N, Eisen T, Maynard J, Bridle H, Shah B, Fleischmann C, Sampson JR, Cheadle JP, Houlston RS (2004) Inherited variants in MYH are unlikely to contribute to the risk of lung carcinoma. Hum Genet 114: $207-210$

Ames BN, Gold LS (1991) Endogenous mutagens and the causes of aging and cancer. Mutat Res 250: 3-16

Croitoru ME, Cleary SP, Di Nicola N, Manno M, Selander T, Aronson M, Redston M, Cotterchio M, Knight J, Gryfe R, Gallinger S (2004) Association between biallelic and monoallelic germline $\mathrm{MYH}$ gene mutations and colorectal cancer risk. J Natl Cancer Inst 96: $1631-1634$

Enholm S, Hienonen T, Suomalainen A, Lipton L, Tomlinson I, Karja V, Eskelinen M, Mecklin JP, Karhu A, Jarvinen HJ, Aaltonen LA (2003) Proportion and phenotype of MYH-associated colorectal neoplasia in a population-based series of Finnish colorectal cancer patients. Am J Pathol 163: $827-832$

Farrington SM, Tenesa A, Barnetson R, Wiltshire A, Prendergast J, Porteous M, Campbell H, Dunlop MG (2005) Germline susceptibility to colorectal cancer due to base-excision repair gene defects. Am J Hum Genet 77: $112-119$

Fleischmann C, Peto J, Cheadle J, Shah B, Sampson J, Houlston RS (2004) Comprehensive analysis of the contribution of germline MYH variation to early-onset colorectal cancer. Int J Cancer 109: 554-558

Higgins JP, Thompson SG (2002) Quantifying heterogeneity in a metaanalysis. Stat Med 21: $1539-1558$
Our study confirms and provides more precise estimates for the homozygous effect and strengthens the evidence for a weak heterozygous effect. The study underscores some of the difficulties of studying the role of low-frequency variants of small effect and emphasises the need for international collaboration to achieve the very large sample sizes required to identify these variants and quantify their effects.

\section{ACKNOWLEDGEMENTS}

We thank Alice Wiltshire for performing the genotyping of the replication cohort. The work is funded by grants from the Chief Scientist Office (CZB/4/94), Cancer Research UK (C348/A3758) and Medical Research Council (G0000657-53203). carriers of MYH mutations: a population-based case-family study. Cancer Epidemiol Biomarkers Prev 15: $312-314$

Kambara T, Whitehall VLJ, Spring KJ, Barker MA, Arnold S, Wynter CVA, Matsubara N, Tanaka N, Young JP, Leggett BA, Jass JR (2004) Role of inherited defects of MYH in the development of sporadic colorectal cancer. Gene Chromosome Cancer 40: 1-9

Peterlongo P, Mitra N, Chuai S, Kirchhoff T, Palmer C, Huang H, Nafa K, Offit K, Ellis NA (2005) Colorectal cancer risk in individuals with biallelic or monoallelic mutations of MYH. Int J Cancer 114: 505-507

Tao H, Shinmura K, Hanaoka T, Natsukawa S, Shaura K, Koizumi Y, Kasuga Y, Ozawa T, Tsujinaka T, Li Z, Yamaguchi S, Yokota J, Sugimura $\mathrm{H}$, Tsugane $\mathrm{S}$ (2004) A novel splice-site variant of the base excision repair gene MYH is associated with production of an aberrant mRNA transcript encoding a truncated MYH protein not localized in the nucleus. Carcinogenesis 25: 1859-1866

Tenesa A, Farrington SM, Dunlop MG (2005) Re: association between biallelic and monoallelic germline MYH gene mutations and colorectal cancer risk. J Natl Cancer Inst 97: 320 - 321

Wang L, Baudhuin LM, Boardman LA, Steenblock KJ, Petersen GM, Halling KC, French AJ, Johnson RA, Burgart LJ, Rabe K, Lindor NM, Thibodeau SN (2004) MYH mutations in patients with attenuated and classic polyposis and with young-onset colorectal cancer without polyps. Gastroenterology 127: 9-16

Yoshimura K, Hanaoka T, Ohnami S, Ohnami S, Kohno T, Liu Y, Yoshida T, Sakamoto H, Tsugane S (2003) Allele frequencies of single nucleotide polymorphisms (SNPs) in 40 candidate genes for gene-environment studies on cancer: data from population-based Japanese random samples. J Hum Genet 48: 654-658

Zhou XL, Djureinovic T, Werelius B, Lindmark G, Sun XF, Lindblom A (2005) Germline mutations in the MYH gene in Swedish familial and sporadic colorectal cancer. Genet Testing 9: 147-151 